\title{
Protein kinase $C-\delta$ is involved in the inflammatory effect of IL-6 in mouse adipose cells
}

\author{
E. Wallerstedt • U. Smith • C. X. Andersson
}

Received: 22 September 2009 / Accepted: 17 December 2009/Published online: 12 February 2010

(C) Springer-Verlag 2010

\begin{abstract}
Aims/hypothesis The aim of the study was to address the role of protein kinase $\mathrm{C}-\delta(\mathrm{PKC} \delta$ ) on phosphorylation of signal transducer and activator of transcription 3 (STAT3) and activation of inflammatory genes in response to IL-6 in adipose cells.

Methods Differentiated mouse 3T3-L1 adipocytes preincubated with the PKC $\delta$ inhibitor rottlerin and mouse embryonic fibroblasts (MEFs) lacking PKC $\delta$ were incubated with IL-6 and/or insulin. RNA was extracted and the gene expression was analysed by real-time PCR, while the proteins from total, nuclear and cytoplasmic lysates were analysed by immunoblotting.

Results Inhibition of PKC $\delta$ by rottlerin significantly reduced both Ser-727 and Tyr-705 phosphorylation of STAT3. Consequently, nuclear translocation of STAT3 and the IL-6-induced gene transcription and protein release of the inflammatory molecule serum amyloid A 3 (SAA3) were reduced. Similarly, the IL-6-regulated gene transcription of $I l-6$ (also known as IlO) to $H p$ and the feedback inhibitor of IL-6, Socs3, were also attenuated by rottlerin. Furthermore, PKC $\delta$ was found to translocate to the nucleus following IL- 6 treatment and this was also reduced by
\end{abstract}

Electronic supplementary material The online version of this article (doi:10.1007/s00125-010-1668-1) contains supplementary material, which is available to authorised users.

E. Wallerstedt · U. Smith · C. X. Andersson $(\bowtie)$

The Lundberg Laboratory for Diabetes Research,

Center of Excellence for Cardiovascular and Metabolic Research,

Department of Molecular and Clinical Medicine/Diabetes,

The Sahlgrenska Academy at University of Gothenburg,

Blå Stråket 5 ,

SE-413 45 Gothenburg, Sweden

e-mail: christian.andersson@gu.se rottlerin. In agreement with the effect of rottlerin, $P k c \delta$ (also known as $P r k c d)^{-/-}$MEFs also displayed a markedly reduced ability of IL-6 to activate the transcription of Saa3, $H p$, Socs 3 and $I l 6$ genes compared with wild-type MEFs. These results correlated with a reduced nuclear translocation and phosphorylation of STAT3.

Conclusions/interpretation These results show that PKC $\delta$ plays a key role in the inflammatory effect of IL-6 in adipose cells and may be a suitable target for novel antiinflammatory agents.

Keywords 3T3-L1 $\cdot$ IL-6 $\cdot$ Inflammation . Insulin resistance $\cdot$ Obesity $\cdot$ PKC delta $\cdot \mathrm{STAT} 3$
Abbreviations
ERK Extracellular signal-regulated kinase
gp130 Glycoprotein 130
Hp Haptoglobin
HRP Horseradish peroxidase
IRS-1 Insulin receptor substrate-1
JAK2 Janus kinase 2
JNK c-Jun N-terminal kinase
MAPK Mitogen-activated protein kinase
MEF Mouse embryonic fibroblast
mTOR Mammalian target of rapamycin
NPM Nucleophosmin
$\mathrm{PKC} \delta \quad$ Protein kinase $\mathrm{C}-\delta$
PLC $\gamma$-1 Phospholipase C $\gamma-1$
RQ Relative quantity
SAA3 Serum amyloid A 3
siRNA Small interfering RNA
SOCS3 Suppressor of cytokine signalling 3
STAT Signal transducer and activator of transcription
WT Wild-type 


\section{Introduction}

The adipose tissue is not only the main organ for lipid storage and release but it also has important paracrine and endocrine functions by secreting different adipokines, including cytokines, chemokines, growth factors and complement factors. The expression of inflammatory molecules is increased in obesity and associated with inflammation in the adipose tissue, which, in turn, negatively affects insulin sensitivity [1]. A major secreted inflammatory cytokine is IL-6 [2]. The interstitial level of IL-6 in the adipose tissue is considerably higher than in the peripheral circulation and correlates with the size of fat cells [3], a marker of local inflammation in adipose tissue. Furthermore, $\sim 30 \%$ of the circulating levels of IL-6 originate from adipose tissue [4].

Chronically elevated IL-6 levels have been shown to negatively affect insulin signalling and action in liver [5], adipose cells [6-8] and skeletal muscles [9]. Important target molecules for IL-6 are peroxisome proliferatoractivated receptor $\gamma$, adiponectin, GLUT4 and insulin receptor substrate-1 (IRS-1) [3, 8, 10]. IL-6 both impairs the transcriptional activation of these molecules and increases the degradation of IRS-1 [11, 12]. One mechanism for this is the IL-6-induced increase of the suppressor of cytokine signalling 3 (SOCS3), a feed-back inhibitor of the signal transducer and activator of transcription (STAT) 3 signalling, which also binds to IRS-1 targeting it for proteasomal degradation. The overall effect of IL-6 is the induction of insulin resistance through an impaired insulin signal transduction [11-13] and decreased insulinstimulated glucose uptake in both adipocytes $[8,14]$ and skeletal muscle [9].

IL-6 signals through a complex of heterodimerised IL-6 and glycoprotein 130 (gp130) receptors. This complex activates tyrosine kinases from the Janus kinase (JAK) family allowing phosphorylation of gp130. STATs, mainly STAT3, bind via the SH2 domain to phosphotyrosine residues on the gp130 receptor, allowing STAT3 to become phosphorylated on Tyr-705. This phosphorylation is necessary for the homo- or heterodimerisation of STAT3 and STAT1, and a prerequisite for the nuclear translocation of the STAT molecules $[15,16]$.

The additional Ser-727 phosphorylation has been reported to increase the transcriptional activation of STAT3 [17-21]. Depending on cell origin, many different kinases have been shown to increase Ser-727 phosphorylation of STAT3, such as protein kinase $\mathrm{C}-\delta$ (PKC $\delta$ ), extracellular signal-regulated kinase (ERK), c-Jun N-terminal kinase (JNK), p38 mitogen-activated protein kinase (MAPK) and the mammalian target of rapamycin (mTOR) [18-22].

We have previously reported that insulin exerts an antiinflammatory effect on the IL-6 signalling pathway by reducing Tyr-705 phosphorylation and nuclear translocation of STAT3, leading to a reduction of IL-6-activated genes. We also showed that insulin increases Ser-727 phosphorylation of STAT3 through a MAPK-dependent pathway that was different from that of IL-6 [23].

In this study, we further characterised Ser- 727 phosphorylation of STAT3 by IL- 6 and its potential role in mouse 3T3-L1 adipocytes by using specific inhibitors for the kinase pathways presented above [18-22]. We concluded that PKC $\delta$ was not only important for Ser-727 phosphorylation, but also for the Tyr-705 phosphorylation, nuclear translocation and transcriptional properties of STAT3. Furthermore, IL-6-inducible inflammatory genes such as Il-6 (also known as $\mathrm{IlO}$ ), serum amyloid A 3 (Saa3) and $\mathrm{Hp}$, as well as the feedback regulator Socs3, were all reduced by inhibition of $\mathrm{PKC} \delta$. This effect appears specific for IL-6induced inflammatory genes, since other non-IL-6-inducible but STAT-regulated genes were not altered.

\section{Methods}

Cell cultures Mouse 3T3-L1 preadipocytes, wild-type (WT) mouse embryonic fibroblasts (MEFs) and $P k c \delta$ (also known as Prkcd) $)^{-/-}$MEFs (a kind gift from M. E. Reyland, University of Colorado Health Sciences Center, Aurora, CO, USA) [24] were grown in DMEM supplemented with $10 \%$ (vol./vol.) FBS, 2 mmol/l glutamine and $1 \%$ penicillin/ streptomycin (1\% [vol./vol.]) [24]. MEFs were also supplemented with non-essential amino acids (Lonza, Rockland, ME, USA). The 3T3-L1 cells were differentiated 2 days after confluence, as described before [23]. On day 8, the differentiated 3T3-L1 adipocytes were starved in DMEM containing $1.5 \%$ (vol./vol.) FBS for $8 \mathrm{~h}$, washed twice with PBS and then incubated with DMEM supplemented with $0.5 \%$ (wt/ vol.) BSA for another $16 \mathrm{~h}$ before starting the experiment on day 9. Insulin (100 nmol/l) was added $10 \mathrm{~min}$ before IL-6 (20 ng/ml; Chemicon, Temecula, CA, USA) while rottlerin $(6 \mu \mathrm{mol} / 1$; Sigma-Aldrich, St Louis, MO, USA) was added $30 \mathrm{~min}$ before addition of IL-6. MEFs were starved for $16 \mathrm{~h}$ in DMEM supplemented with $0.5 \%$ (wt/vol.) BSA before addition of $30 \mathrm{ng} / \mathrm{ml}$ IL- 6 together with $30 \mathrm{ng} / \mathrm{ml}$ soluble IL6 receptor (Sigma-Aldrich) as indicated. Transfection of PKCS small interfering RNA (siRNA; Sigma-Aldrich) in 3T3-L1 cells was performed on day 3 in the differentiation cycle using Liposome 2000 transfection reagent (Invitrogen, Carlsbad, CA, USA).

Cell lysates and immunoblotting Differentiated 3T3-L1 adipocytes were cultured in $25 \mathrm{~cm}^{2}$ flasks, washed twice with ice-cold PBS and lysed in lysis buffer as previously described [23]. The cell lysate was centrifuged at 20,000 $\times g$ for $10 \mathrm{~min}$ and the supernatant fraction was kept at $-80^{\circ} \mathrm{C}$. 
The nuclear cell extract was prepared as suggested by the manufacturer of the STAT3 transcription factor assay kit (Active Motif, Carlsbad, CA, USA). Protein concentration was quantified by the bicinchonic acid method (Pierce, Rockford, IL) or a Bradford protein assay kit (Bio-Rad Laboratories, Hercules, CA, USA). The lysates were boiled in SDS loading buffer for $5 \mathrm{~min}$ and applied to SDS-PAGE (Lonza, Rockland, ME, USA). Following gel electrophoresis and gel transference, the nitrocellulose membranes were blocked for $1 \mathrm{~h}$ with $5 \%$ (wt/vol.) fat-free milk powder diluted in PBS-TWEEN 20 (0.05\% vol./vol.). Membranes were probed with primary antibodies to the following proteins: Tyr-705-phosphorylated STAT3, Tyr-701phosphorylated STAT1, nucleophosmin (NPM) and PKC (Cell Signaling Technologies, Beverly, MA, USA), Ser-727phosphorylated STAT3 and gp130 (Santa Cruz Biotechnology, Santa Cruz, CA, USA), phospholipase C (PLC) $\gamma-1$ (Upstate Biotechnology, Lake Placid, NY, USA), STAT3 (BD Biosciences, San Jose, CA, USA) and murine SAA3 (kind gift from P. Scherer, University of Texas Southwestern Medical Center, Dallas, TX, USA). Antibodies were diluted according to the recommendation of the manufacturers. The blots were probed with secondary antibody linked to horseradish peroxidase (HRP; Cell Signaling) diluted 1:1,000 in blocking solution. Detection was with an Immun-STAR HRP chemiluminescence kit using a ChemiDoc XRS detection system (Bio-Rad).

STAT3 transcription assay Nuclear extracts were prepared and analysed on a 96 well plate coated with oligonucleotides corresponding to the STAT3 consensus binding site. STAT3 primary antibody was added to the plate followed by HRP-conjugated secondary antibody. The absorbance was read on a spectrophotometer. Thus, this kit measures nuclear STAT3 binding to its consensus binding site. The protocol was essentially performed as suggested by the manufacturer of the STAT3 transcription factor assay (Active Motif).

$R T-P C R$ and quantitative real-time PCR Cells were washed in RNAse-free PBS and mRNA was extracted using the RNeasy Mini Protocol (Qiagen, Valencia, CA, USA). cDNA was synthesised from $0.4 \mu \mathrm{g}$ total RNA using the High Capacity cDNA Reverse Transcriptase kit (Applied Biosystems, Foster City, CA, USA). The samples were quantified using an ABI Prism 7900HT Sequence Detection System (Applied Biosystems) using gene-specific primers and probes. The sequences are available upon request. $18 \mathrm{~S}$ ribosomal RNA was used as an endogenous control for each sample.

Statistical analyses Statistical analyses were performed in SPSS or Microsoft Excel with a paired Wilcoxon test, unpaired Mann-Whitney test or paired and unpaired Student's $t$ tests as indicated in figure legends. $p<0.05$ was considered statistically significant. Results shown in the figures are means \pm SEM.

\section{Results}

Inhibition of PKC $\delta$ with rottlerin inhibits STAT3 signalling We have previously shown that incubation of differentiated 3T3-L1 cells with insulin or IL-6 increases Ser-727 phosphorylation of STAT3 through different protein kinases [23]. An extensive search for known potential kinases inducing Ser-727 phosphorylation of STAT3 in response to IL-6 was performed by using inhibitors to PKC $\delta$, ERK, JNK, p38 MAPK and mTOR (data not shown), as they have previously been described to induce this phosphorylation [18-22]. Our study revealed PKC $\delta$ as the main target molecule. We then analysed the effect of the PKC $\delta$ inhibitor, rottlerin, on STAT3 activation. Pretreatment with rottlerin significantly reduced the IL-6-induced Ser727 phosphorylation of STAT3, whereas no significant effect was seen on the insulin-induced phosphorylation (Fig. 1a, c). We also examined the effect of rottlerin on Tyr705 phosphorylation of STAT3; pretreatment with rottlerin for 30 min significantly inhibited this phosphorylation (Fig. 1d, f), while total STAT3 was not altered in the experiments (Fig. 1b, e).

Rottlerin reduces the transcriptional effect of IL-6 on inflammatory genes We have previously reported that IL6 activates the transcription of Socs $3, S a a 3$ and $H p$ genes in differentiated 3T3-L1 adipocytes [23]. Pretreatment with rottlerin significantly reduced the IL-6-induced activation of Socs 3 and the $I l-6$ gene itself $(1 \mathrm{~h})$, as well as of $H p$ and Saa3 (24 h; Fig. 2a). Another general PKC inhibitor, H7, also totally blocked the activation of these genes by IL-6 (Electronic supplementary material [ESM] Fig. 1). However, other non-IL-6-inducible STAT-regulated genes, such as Pepck (also known as Pckl) or G6pc [25], were not significantly altered (Fig. 2b), suggesting that PKC $\delta$ is particularly important for the activation of inflammatory genes by IL- 6 .

Secreted SAA3 protein to the culture medium was also analysed by immunoblotting. Culture media from differentiated 3T3-L1 cells incubated for $24 \mathrm{~h}$ with IL-6 alone displayed an increased secretion of SAA3, whereas this was inhibited by rottlerin (Fig. 2c, d).

Effect of rottlerin on the transcriptional properties and nuclear localisation of STAT3 As rottlerin markedly impaired IL-6-induced STAT3 phosphorylation and gene activation, we also investigated the effect of rottlerin on 
Fig. 1 Effect of rottlerin on STAT3 phosphorylation. Immunoblots of lysates from differentiated 3T3-L1 adipose cells pretreated with rottlerin before addition of insulin and/or IL-6 for $30 \mathrm{~min}$. Immunoblots showing Ser-727phosphorylated STAT3 (pS-STAT3) (a) and total STAT3 protein (b). c Bar chart of the corresponding relative band intensities (Rel. intens.) from five individual experiments (means \pm SEM, basal $=1$, ${ }^{*} p<0.05$, paired Student's $t$ test). Immunoblots showing Tyr-705-phosphorylated STAT3 (pY-STAT3) (d) and total STAT3 (e). f Bar chart of the corresponding band intensities from six individual experiments (means \pm SEM, basal $=1$, $* * p<0.01$, paired Student's $t$ test). Bas, basal; ins, insulin; rott., rottlerin

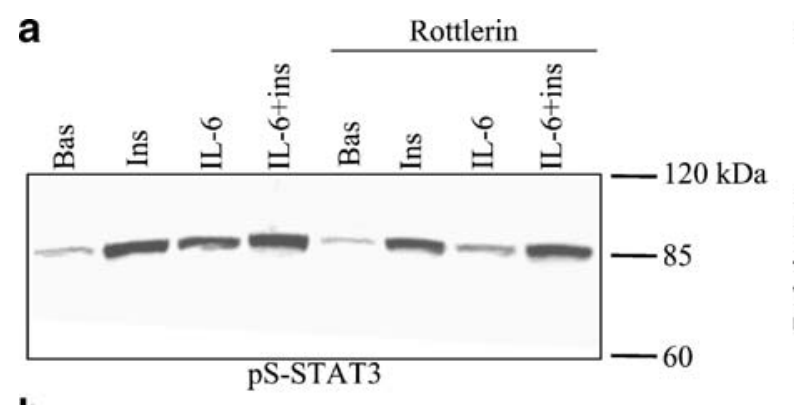

b

C

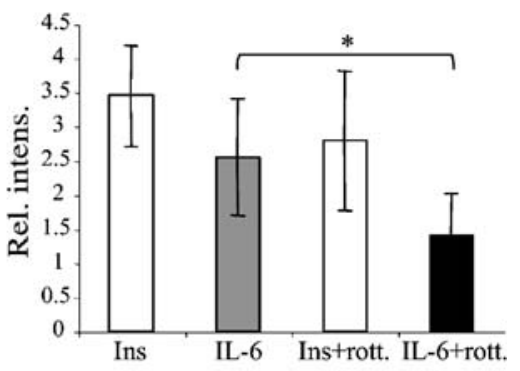

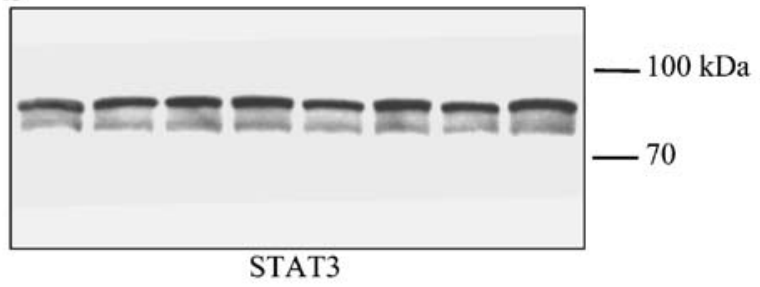

d
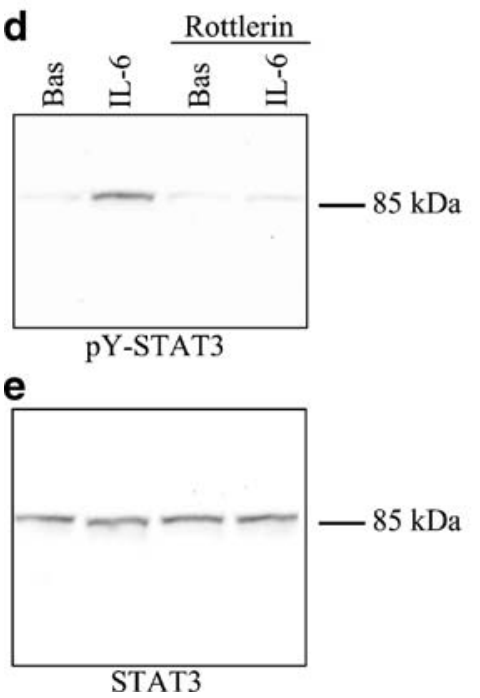

f

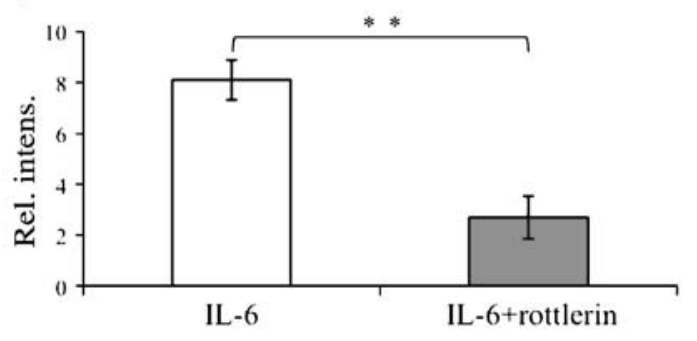

STAT3 nuclear translocation and its transcriptional properties (STAT3 binding to consensus sequence). Differentiated 3T3-L1 cells, pretreated with rottlerin before IL-6 addition, showed $42 \%(p<0.05)$ reduced DNA binding of STAT3 compared with IL-6 alone (Fig. 3a). Furthermore, nuclear translocation of STAT3 was also markedly reduced (Fig. 3b, c), which is consistent with the inhibitory effect of rottlerin on STAT3 phosphorylation. In agreement with Fig. 1a, Ser-727-phosphorylated STAT3 in the nucleus also appeared to be reduced in the presence of rottlerin, although this difference did not reach statistical significance (ESM Fig. 2a).

Rottlerin reduces the nuclear translocation of PKC $\delta$ in 3T3-L1 cells To investigate the potential co-localisation of PKC $\delta$ with STAT3, we examined nuclear extracts from differentiated 3T3-L1 adipocytes and found that nuclear translocation of PKC $\delta$ was increased in response to IL-6 and that this could be inhibited by the addition of rottlerin
(Fig. 3d, e). At this time point, STAT3 is also translocated to the nucleus following IL-6 (Fig. 3b).

Reduced effect of IL-6-induced gene expression in $P k c \delta^{-1}$ $M E F s$ We also investigated the role of PKC $\delta$ in the IL-6induced inflammatory response in $P k c \delta^{-/}$MEF cells. IL-6 alone increased Socs 3 and Il-6 mRNA levels after $1 \mathrm{~h}$ in both cell types (Fig. 4). However, the inductions of these genes were much lower $(\sim 80 \%)$ in $P k c \delta^{/-}$MEFs compared with WT MEFs (Fig. 4). Furthermore, in contrast to WT MEFs, $P k c \delta^{-/}$cells did not increase gene transcription of $H p$ or Saa3 when stimulated with IL-6 for 24 h (Fig. 4). These findings further support a critical role for $\mathrm{PKC} \delta$ in STAT3 activation of inflammatory genes in response to IL6. In addition we attempted to transfect differentiated 3T3L1 cells with PKC $\delta$-siRNA. We were able to downregulate $\sim 25 \%$ of the PKC $\delta$ expression when cells were transfected on the third day in the differentiation cycle (ESM Fig. 3). On day 8 , cells were incubated with IL-6 for $24 \mathrm{~h}$ followed 

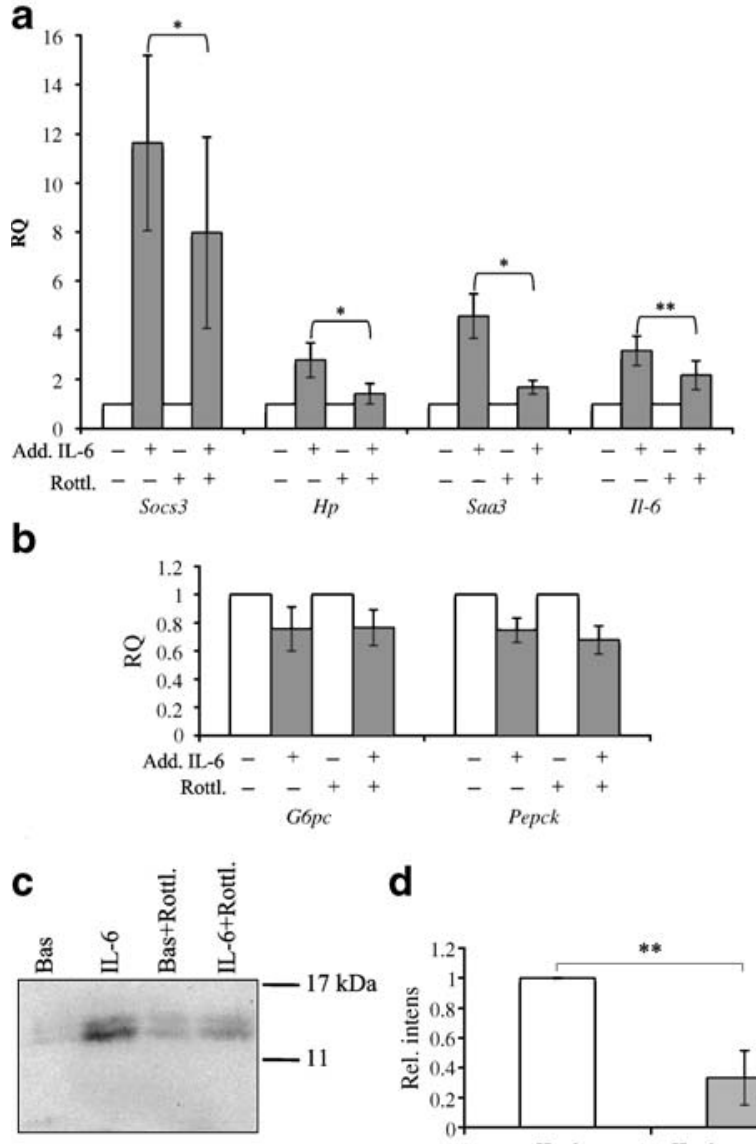

d

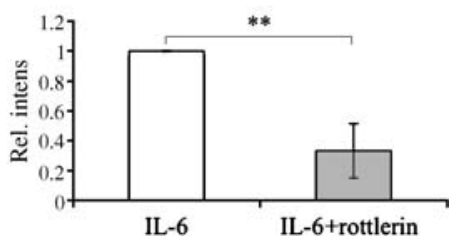

Fig. 2 The effect of rottlerin on IL-6-induced mRNA expression was measured by real-time PCR (white bars, basal samples; grey bars, IL-6 samples). a mRNA expression of IL-6-activated genes. Differentiated 3T3-L1 adipose cells were pretreated with rottlerin before incubation with IL-6. The mRNA levels of Socs 3 and Il-6 were analysed $1 \mathrm{~h}$ after IL-6 addition (Add.) ( $n=6$ experiments) and $H p$ and Saa3 were analysed $24 \mathrm{~h}$ after IL- 6 addition ( $n=6$ experiments). Means \pm SEM, $* p<0.05$, paired Wilcoxon test. b Control experiment showing no alteration (paired Student's $t$ test) of mRNA levels of the noninflammatory STAT3-regulated genes G6pc and Pepck (means \pm SEM, $n=4$ experiments) when pretreated with rottlerin and subjected to IL-6 for $1 \mathrm{~h}$. c Immunoblot showing secreted SAA3 protein in culture media from differentiated 3T3-L1 cells incubated with IL-6 for $24 \mathrm{~h}$ with or without rottlerin. The bar chart (d) shows corresponding relative band intensities (Rel. Intens.) from four individual experiments, IL-6 samples are set to 1 . Means \pm SEM, $* * p<0.01$, paired Student's $t$ test. Bas, basal; Rottl., rottlerin

by RNA preparation. Real-time PCR analysis of the IL-6induced gene expression of $\mathrm{Saa} 3$ and $\mathrm{Hp}$ also showed lower expression of these genes in PKC $\delta$-siRNAtransfected cells compared with non-transfected cells (ESM Fig. 3). However, owing to large variation this reduction did not reach statistical significance.

Reduced nuclear localisation of STAT3 in Pkc $\delta^{-/-}$ MEFs We also analysed the nuclear translocation of STAT3 in $P k c \delta^{--}$MEFs. Similar to the findings in 3T3-L1 cells incubated with rottlerin (Fig. 3b), nuclear translocation of STAT3 in response to IL-6 was markedly reduced in $P k c \delta^{-1}$ MEFs (Fig. 5a, b). The reduced nuclear localisation of STAT3 was also associated with a decreased Tyr-705

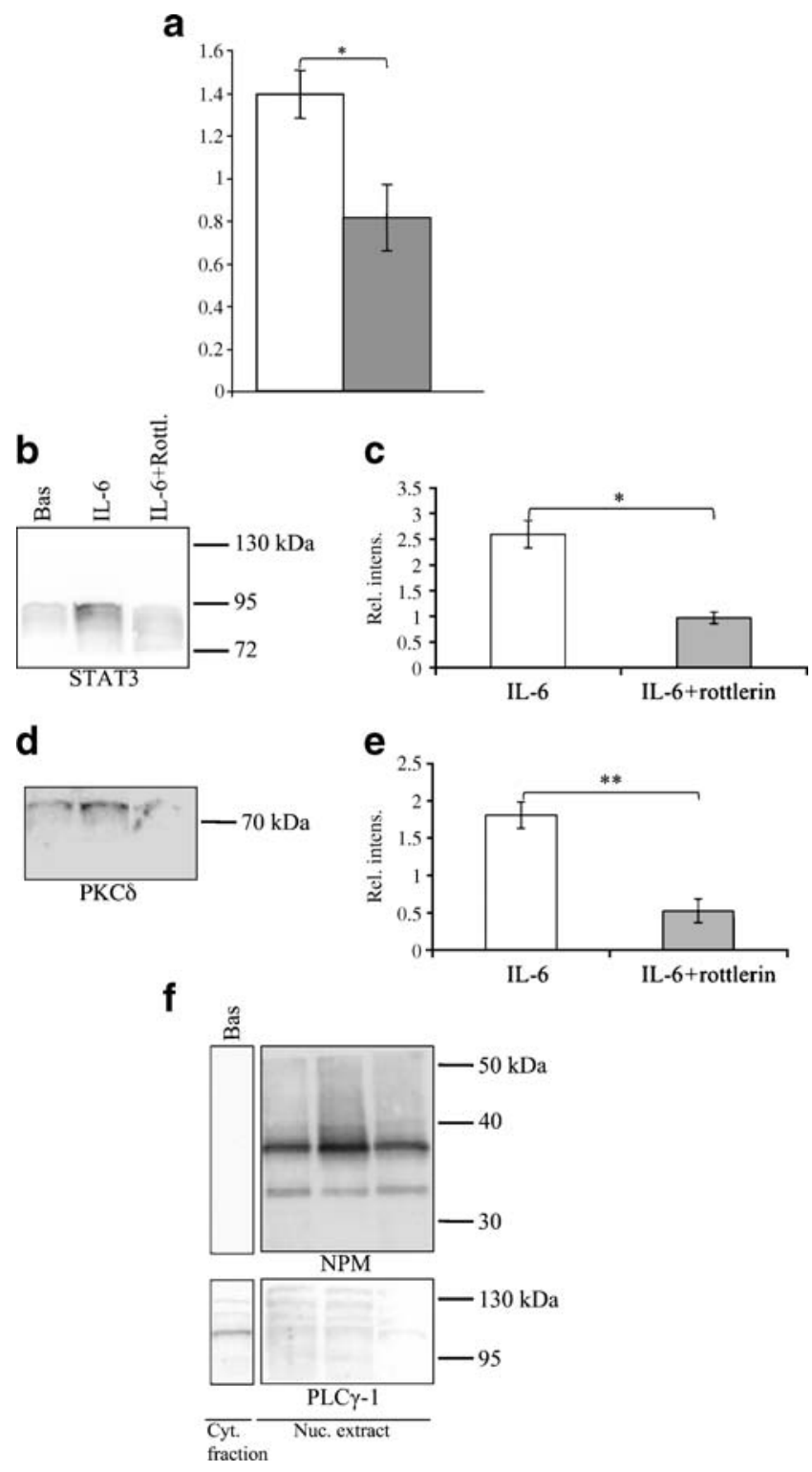

Fig. 3 Effect of rottlerin on transcriptional activation properties and nuclear localisation of STAT3 (white bars, basal samples; grey bars, IL-6 samples). a Transcriptional activity of STAT3. Bar chart showing the ability of nuclear STAT3 to bind to its consensus sequence in samples pretreated with rottlerin and incubated with IL-6 for $15 \mathrm{~min}$ ( $n=6$ experiments). Means \pm SEM, ${ }^{*} p<0.05$, paired Wilcoxon test. b-e Nuclear localisation of STAT3 and PKC $\delta$. Immunoblots showing STAT3 (b) and PKC $\delta$ (d) in nuclear lysates from differentiated 3T3L1 adipose cells incubated with IL- 6 or IL- $6+$ rottlerin for $15 \mathrm{~min}$ and bar charts of the average corresponding relative band intensities (Rel. intens.) for STAT3 (c) $(n=3)$ and PKC $\delta(\mathbf{e})(n=5)$. Means \pm SEM, ${ }^{*} p<$ $0.05,{ }^{* *} p<0.01$, paired Student's $t$ test. f Blots showing NPM and PLC $\gamma-1$ used as nuclear (Nuc.) and cytoplasmic (Cyt.) markers, respectively. Bas, basal; Rel. intens., relative intensity (basal sample set to 1); Rottl., rottlerin 


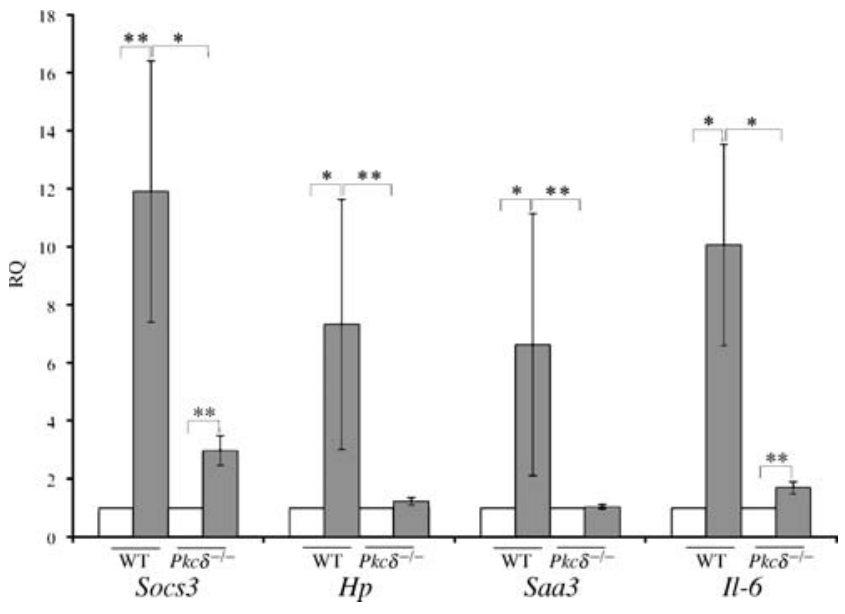

Fig. 4 Reduced effect of IL-6 in $P k c \delta^{-/}$MEFs. WT MEFs and $P k c \delta^{-/-}$MEFs incubated with IL-6 (white bars, basal samples; grey bars, IL-6 samples). The mRNA levels of Socs 3 and Il-6 after $1 \mathrm{~h}$, and of $\mathrm{Hp}$ and Saa3 after $24 \mathrm{~h}$, were measured by real-time PCR. Results are means \pm SEM of 6-12 individual experiments. ${ }^{*} p<0.05,{ }^{* *} p<0.01$; paired Wilcoxon test for basal-IL-6 samples and unpaired MannWhitney test for IL-6-IL-6 samples

phosphorylation in response to IL-6 in $P k c \delta^{-/}$cells (Fig. 5a, b). As for rottlerin-treated adipocytes, IL-6induced Ser-727 phosphorylation of nuclear STAT3 also tended to be reduced, but the difference did not reach statistical significance (ESM Fig. 2b). Lysates from the cytoplasmic fraction were also analysed by immunoblotting using the same STAT3 antibody. In WT MEFs, cytoplasmic

a

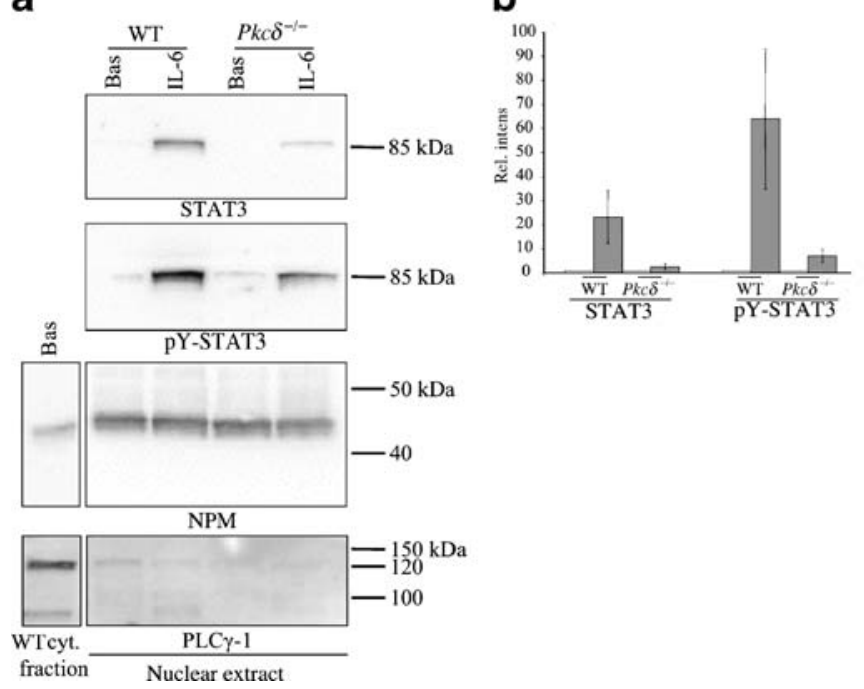

STAT3 protein levels in response to IL-6 were reduced, but this was not seen in $P k c \delta^{-/}$MEFs (Fig. 5c, d). This correlates with the STAT3 translocation seen in the nuclear extracts (Fig. 5a).

\section{Discussion}

As for humans, genetic variations between different mice strains are associated with a differential sensitivity to the complications of obesity. In a comparison between $\mathrm{C} 57 \mathrm{Bl} / 6$ (B6) mice and 129S6/SvEvTac, the B6 mice were more sensitive to becoming obese, insulin resistant and glucose intolerant, independently of the fat content in the food. Interestingly, these mice also displayed an upregulation of PKC $\delta$ in skeletal muscle, liver and, especially, in epididymal fat [26]. Interestingly, it has recently been shown that PKC 8 -deficient mice fed on high-fat diet displayed improved glucose tolerance because of increased insulin sensitivity. Same mice also showed reduced levels of triacylglycerol in the liver [27]. In another study, isolated adipocytes from B6 mice fed a high-fat diet showed impaired insulin-induced glucose uptake, oxidative stress and activation of $\mathrm{PKC} \delta$, which were suggested to be important for obesity-induced insulin resistance in the adipose tissue [28]. It has also been shown that PKC $\delta$ is involved in insulin-induced Ser-318 phosphorylation of IRS-1, thus impairing insulin signalling [29].
C

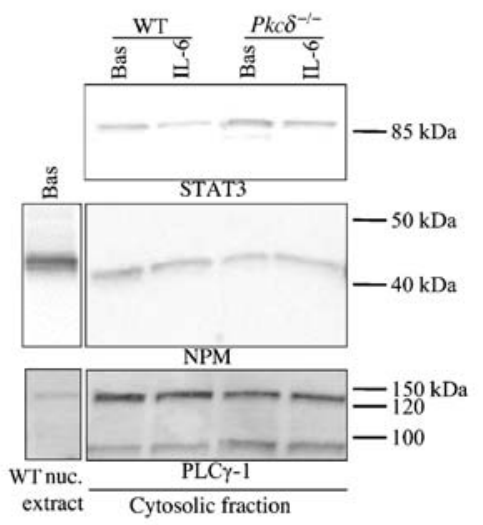

d

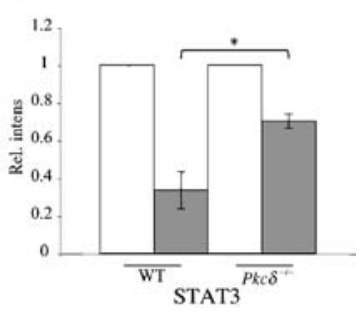

and a bar chart (d) of corresponding relative band intensities (Rel. intens.) from WT MEFs and $P k c \delta^{-/}$MEFs (white bars, basal samples; grey bars, IL-6 samples) incubated with IL-6 for $15 \mathrm{~min}$ $\left(n=3\right.$; basal is set to 1 for each cell type). Means \pm SEM, ${ }^{*} p<0.05$, unpaired Student's $t$ test. Blots showing NPM and PLC $\gamma-1$ are used as nuclear (nuc.) and cytoplasmic (cyt.) markers, respectively (a, c)
Fig. 5 Reduced nuclear translocation of STAT3 in $P k c \delta^{-/}$MEFs. Immunoblots showing STAT3 and Tyr-705-phosphorylated STAT3 (pY-STAT3) in nuclear lysates (a) and a bar chart (b, means \pm SEM) of the corresponding relative band intensities (Rel. intens.) for nuclear STAT3 and Tyr-705-phosphorylated STAT3 ( $n=3$ experiments) for WT MEFs and $P k c \delta^{-1}$ MEFs samples incubated with IL-6 for 15 min. Immunoblots showing STAT3 in the cytoplasmic fraction (c) 
In this study, the role of several kinases (e.g. PKC $\delta$, ERK, JNK, p38 MAPK and mTOR) on IL-6 signalling and activation of inflammatory genes in 3T3-L1 adipose cells was examined. We found that $\mathrm{PKC} \delta$ plays a critical role in STAT-3 signalling in these cells, since the addition of rottlerin inhibited: (1) IL-6-induced Tyr-705 and Ser-727 phosphorylation of STAT3; (2) nuclear translocation and transcriptional binding of STAT3; (3) IL-6-induced activation of inflammatory genes like $\mathrm{Il}-6, \mathrm{Saa} 3$ and $\mathrm{Hp}$, as well as the feed-back inhibitor Socs3; and (4) SAA3 secretion to the culture medium. Although rottlerin has been claimed to be a specific inhibitor of PKC $\delta$ [30], recent studies have shown that it may have additional effects in the mitochondria and on 5'-AMP-activated protein kinase [31, 32]. Therefore, we further corroborated our findings in 3T3-L1 cells with rottlerin by using $P k c \delta^{-/}$MEFs. Essentially all results seen with rottlerin and IL-6 in 3T3-L1 adipocytes were confirmed in $P k c \delta^{-/}$MEFs. Additionally, we were able to attenuate the expression of PKC $\delta$ using siRNA, which also tended to decrease the IL-6-induced gene transcription of $S a a 3$ and $H p$, further supporting a role for PKC $\delta$ in IL-6 signalling. Interestingly, we found in both $P k c \delta^{-1}$ MEFs and 3T3-L1 adipose cells incubated with rottlerin that the effect of IL-6 on Tyr-705 phosphorylation and on Ser-727 phosphorylation of STAT3 was reduced. Together, these results show that PKC $\delta$ plays a key role in IL-6-induced STAT3 phosphorylation and activation. Tyr705 phosphorylation is crucial for dimerisation and nuclear translocation of STAT3 [15]. Thus, the reduced nuclear translocation of STAT3 seen in the presence of rottlerin and in $P k c \delta^{-1}$ MEFs is an expected consequence of the reduced tyrosine phosphorylation.

There are few reports focusing on the effect of rottlerin on the Tyr-705 phosphorylation of STAT3. In HepG2 cells, Schuringa et al. [18] found no inhibition of IL-6-induced STAT3 Tyr-705 phosphorylation while Xu et al. [33] found that pretreatment with rottlerin totally blocked IL-13-induced STAT3-DNA binding in monocytes. Another report showed that prolactin- or rat placental lactogen 1-induced Tyr-705 phosphorylation of STAT3 is reduced in response to rottlerin in granulosa cells and, thus, also STAT3-DNA binding [34].

PKC $\delta$ is obviously involved in (a) critical site(s) of STAT3 activation in response to IL-6. It may play a role in JAK2 activation by IL-6, and this suggestion is supported by a previous report showing that rottlerin reduced the activation of JAK2 in NCM460-NK-1R cells [35]. However, we did not find any difference in JAK2 phosphorylation in the presence or absence of rottlerin (data not shown). Another possibility is that PKC $\delta$ is important for stabilising STAT3 binding to gp130. Reports have shown that PKC $\delta$ associates with Tyr705-phosphorylated STAT3 in the cytoplasm $[22,36]$ and that it can also bind and phosphorylate gp130 [37]. We have also detected an interaction between STAT3 and PKC $\delta$ by co-immunoprecipitation, but this interaction was not altered by either IL-6 or rottlerin (ESM Fig. 4a). Nor did rottlerin interfere with the formation of the STAT3-gp130 complex, but the IL-6-induced tyrosine phosphorylation of STAT3 in this complex was reduced by the presence of rottlerin (ESM Fig. 4b), suggesting that inhibition of PKC $\delta$ affects an upstream kinase. Furthermore, as both STAT1 and STAT3 are similarly activated [15], we analysed IL-6-induced activation of STAT1 and found a small (but detectable) phosphorylation of Tyr-701 (ESM Fig. 5). This phosphorylation was also reduced in the presence of rottlerin, which further supports a role for PKC $\delta$ on an upstream kinase(s).

In order to elucidate if IL- 6 activates PKC $\delta$ by phosphorylation, the PKC $\delta$ phosphorylation sites Thr-505, Ser-643 and Tyr-311 were examined by immunoblotting. However, we could not detect an IL-6-dependent alteration in any of these sites (data not shown). There are very few reports describing an IL-6-induced phosphorylation of PKC $\delta$. Schuringa et al. [18] showed in HepG2 cells that PKC $\delta$ Thr-505 is already phosphorylated in the nucleus 2 min after IL-6 addition. However, we were not able to confirm these results in differentiated 3T3-L1 cells.

Nuclear translocation of $\mathrm{PKC} \delta$ has also been previously studied in HepG2 cells. Schuringa et al. [18] showed that PKC $\delta$ was present in the nucleus independently of IL-6 addition, whereas Jain et al. [22] detected PKC $\delta$ exclusively in the cytoplasm and not in the nucleus in the HepG2 cell line.

We found that PKC $\delta$ is translocated to the nucleus in an IL-6-dependent manner in differentiated 3T3-L1 cells, implying a role for PKC $\delta$ in the transcriptional activation of IL-6-inducible genes. Rottlerin reduced the nuclear colocalisation of PKC $\delta$ and STAT3 as well as the transcription and secretion of IL-6-regulated inflammatory genes. This further supports our concept that $\mathrm{PKC} \delta$ is important for IL6 signal transduction and activation of gene transcription. However, the mRNA levels of several STAT3-regulated but non-IL-6-inducible genes, like Pepck and G6pc were not changed, suggesting that $\mathrm{PKC} \delta$ is particularly important for IL-6-inducible inflammatory genes.

In summary, we here demonstrate that PKC $\delta$ is important for IL-6-induced Tyr-705 and Ser-727 phosphorylation of STAT3, and nuclear translocation, as well as the activation of genes associated with inflammation in 3T3-L1 adipose cells. Our finding that PKC $\delta$ is translocated to the nucleus in response to IL-6, like STAT3, further supports the importance of $\mathrm{PKC} \delta$ as a regulator of this pathway. These findings suggest that $\mathrm{PKC} \delta$ is a potential target for the development of anti-inflammatory agents focusing on the effect of IL-6.

Acknowledgements This study was supported by grants from the European Commission (HEPADIP LSHM-CT-2005-018734), the Swedish Diabetes Association, the Swedish Research Council, the 
Novo Nordisk Foundation, the Sonya Hedenbratt Memorial Fund, the Martina and Willhelm Lundgren Foundation, the IngaBritt and Arne Lundberg Foundation, the Torsten and Ragnar Söderberg's Foundation, the Gothenburg Royal Society of Arts, the Swedish Foundation for Strategic Research, the Lars Hierta Memorial Fund, the O.E. and Edla Johansson Foundation, the Swedish Insurance Society, the Thuring Foundation, the Magn Bergvall Foundation, the Åke Wiberg Foundation and the Gothenburg Medical Society.

Duality of interest The authors declare that there is no duality of interest associated with this manuscript.

\section{References}

1. Gustafson B, Hammarstedt A, Andersson CX, Smith U (2007) Inflamed adipose tissue: a culprit underlying the metabolic syndrome and atherosclerosis. Arterioscler Thromb Vasc Biol 27:2276-2283

2. Pickup JC, Mattock MB, Chusney GD, Burt D (1997) NIDDM as a disease of the innate immune system: association of acute-phase reactants and interleukin- 6 with metabolic syndrome X. Diabetologia 40:1286-1292

3. Sopasakis VR, Sandqvist M, Gustafson B et al (2004) High local concentrations and effects on differentiation implicate interleukin6 as a paracrine regulator. Obes Res 12:454-460

4. Mohamed-Ali V, Goodrick S, Rawesh A et al (1997) Subcutaneous adipose tissue releases interleukin-6, but not tumor necrosis factoralpha, in vivo. J Clin Endocrinol Metab 82:4196-4200

5. Senn JJ, Klover PJ, Nowak IA, Mooney RA (2002) Interleukin-6 induces cellular insulin resistance in hepatocytes. Diabetes 51:3391-3399

6. Bastard JP, Maachi M, van Nhieu JT et al (2002) Adipose tissue IL-6 content correlates with resistance to insulin activation of glucose uptake both in vivo and in vitro. J Clin Endocrinol Metab 87:2084-2089

7. Kern PA, Ranganathan S, Li C, Wood L, Ranganathan G (2001) Adipose tissue tumor necrosis factor and interleukin- 6 expression in human obesity and insulin resistance. Am J Physiol Endocrinol Metab 280:E745-E751

8. Rotter V, Nagaev I, Smith U (2003) Interleukin-6 (IL-6) induces insulin resistance in 3T3-L1 adipocytes and is, like IL-8 and tumor necrosis factor-alpha, overexpressed in human fat cells from insulin-resistant subjects. J Biol Chem 278:45777-45784

9. Franckhauser S, Elias I, Rotter Sopasakis V et al (2008) Overexpression of I16 leads to hyperinsulinaemia, liver inflammation and reduced body weight in mice. Diabetologia 51:1306-1316

10. Fasshauer M, Kralisch S, Klier M et al (2003) Adiponectin gene expression and secretion is inhibited by interleukin-6 in 3T3-L1 adipocytes. Biochem Biophys Res Commun 301:1045-1050

11. Emanuelli B, Peraldi P, Filloux C, Sawka-Verhelle D, Hilton D, van Obberghen E (2000) SOCS-3 is an insulin-induced negative regulator of insulin signaling. J Biol Chem 275:15985-15991

12. Rui L, Yuan M, Frantz D, Shoelson S, White MF (2002) SOCS-1 and SOCS-3 block insulin signaling by ubiquitin-mediated degradation of IRS1 and IRS2. J Biol Chem 277:42394-42398

13. Ueki K, Kondo T, Kahn CR (2004) Suppressor of cytokine signaling 1 (SOCS-1) and SOCS-3 cause insulin resistance through inhibition of tyrosine phosphorylation of insulin receptor substrate proteins by discrete mechanisms. Mol Cell Biol 24:5434-5446

14. Shi H, Cave B, Inouye K, Bjorbaek C, Flier JS (2006) Overexpression of suppressor of cytokine signaling 3 in adipose tissue causes local but not systemic insulin resistance. Diabetes 55:699-707
15. Heinrich PC, Behrmann I, Haan S, Hermanns HM, Muller-Newen G, Schaper F (2003) Principles of interleukin (IL)-6-type cytokine signalling and its regulation. Biochem $\mathrm{J}$ 374:1-20

16. Heinrich PC, Behrmann I, Muller-Newen G, Schaper F, Graeve L (1998) Interleukin-6-type cytokine signalling through the gp130/ Jak/STAT pathway. Biochem J 334:297-314

17. Zhang X, Blenis J, Li HC, Schindler C, Chen-Kiang S (1995) Requirement of serine phosphorylation for formation of STATpromoter complexes. Science 267:1990-1994

18. Schuringa JJ, Dekker LV, Vellenga E, Kruijer W (2001) Sequential activation of Rac-1, SEK-1/MKK-4, and protein kinase Cdelta is required for interleukin-6-induced STAT3 Ser-727 phosphorylation and transactivation. J Biol Chem 276: 27709-27715

19. Wen Z, Zhong Z, Darnell JE Jr (1995) Maximal activation of transcription by Stat 1 and Stat 3 requires both tyrosine and serine phosphorylation. Cell 82:241-250

20. Turkson J, Bowman T, Adnane J et al (1999) Requirement for Ras/Rac1-mediated p38 and c-Jun N-terminal kinase signaling in Stat 3 transcriptional activity induced by the Src oncoprotein. Mol Cell Biol 19:7519-7528

21. Abe K, Hirai M, Mizuno K et al (2001) The YXXQ motif in gp 130 is crucial for STAT3 phosphorylation at Ser727 through an H7-sensitive kinase pathway. Oncogene 20:3464-3474

22. Jain N, Zhang T, Kee WH, Li W, Cao X (1999) Protein kinase C delta associates with and phosphorylates Stat3 in an interleukin-6dependent manner. J Biol Chem 274:24392-24400

23. Andersson CX, Sopasakis VR, Wallerstedt E, Smith U (2007) Insulin antagonizes interleukin-6 signaling and is anti-inflammatory in 3T3-L1 adipocytes. J Biol Chem 282:9430-9435

24. Jackson D, Zheng Y, Lyo D et al (2005) Suppression of cell migration by protein kinase Cdelta. Oncogene 24:3067-3072

25. Ramadoss P, Unger-Smith NE, Lam FS, Hollenberg AN (2009) STAT3 targets the regulatory regions of gluconeogenic genes in vivo. Mol Endocrinol 23:827-837

26. Almind K, Kahn CR (2004) Genetic determinants of energy expenditure and insulin resistance in diet-induced obesity in mice. Diabetes 53:3274-3285

27. Frangioudakis G, Burchfield JG, Narasimhan S et al. (2009) Diverse roles for protein kinase $\mathrm{C}$ delta and protein kinase $\mathrm{C}$ epsilon in the generation of high-fat-diet-induced glucose intolerance in mice: regulation of lipogenesis by protein kinase $\mathrm{C}$ delta. Diabetologia 52:2616-2620

28. Talior I, Yarkoni M, Bashan N, Eldar-Finkelman H (2003) Increased glucose uptake promotes oxidative stress and PKCdelta activation in adipocytes of obese, insulin-resistant mice. Am J Physiol Endocrinol Metab 285:E295-E302

29. Hennige AM, Stefan N, Kapp K et al (2006) Leptin downregulates insulin action through phosphorylation of serine-318 in insulin receptor substrate 1. FASEB J 20:1206-1208

30. Gschwendt M, Muller HJ, Kielbassa K et al (1994) Rottlerin, a novel protein kinase inhibitor. Biochem Biophys Res Commun 199:93-98

31. Soltoff SP (2001) Rottlerin is a mitochondrial uncoupler that decreases cellular ATP levels and indirectly blocks protein kinase Cdelta tyrosine phosphorylation. J Biol Chem 276:37986-37992

32. Soltoff SP (2007) Rottlerin: an inappropriate and ineffective inhibitor of PKCdelta. Trends Pharmacol Sci 28:453-458

33. Xu B, Bhattacharjee A, Roy B, Feldman GM, Cathcart MK (2004) Role of protein kinase C isoforms in the regulation of interleukin-13-induced 15-lipoxygenase gene expression in human monocytes. J Biol Chem 279:15954-15960

34. Peters CA, Maizels ET, Robertson MC, Shiu RP, Soloff MS, Hunzicker-Dunn M (2000) Induction of relaxin messenger RNA expression in response to prolactin receptor activation requires protein kinase C delta signaling. Mol Endocrinol 14:576-590 
35. Koon HW, Zhao D, Zhan Y, Rhee SH, Moyer MP, Pothoulakis C (2006) Substance P stimulates cyclooxygenase-2 and prostaglandin E2 expression through JAK-STAT activation in human colonic epithelial cells. J Immunol 176:5050-5059

36. Bhattacharjee A, Xu B, Frank DA, Feldman GM, Cathcart MK (2006) Monocyte 15-lipoxygenase expression is regulated by a novel cytosolic signaling complex with protein kinase $\mathrm{C}$ delta and tyrosine-phosphorylated Stat3. J Immunol 177:3771-3781

37. Novotny-Diermayr V, Zhang T, Gu L, Cao X (2002) Protein kinase $\mathrm{C}$ delta associates with the interleukin-6 receptor subunit glycoprotein (gp) 130 via Stat3 and enhances Stat3-gp130 interaction. J Biol Chem 277:49134-49142 